\title{
The use of hippocampal volumetric measurements to improve diagnostic accuracy in pediatric patients with mesial temporal sclerosis
}

\author{
Gloria J. Guzmán Pérez-Carrillo, MD, MSc, ${ }^{1}$ Christopher Owen, MA, ${ }^{2}$ \\ Katherine E. Schwetye, MD, PhD, ${ }^{3}$ Spencer McFarlane, BS, ${ }^{2}$ Ananth K. Vellimana, MD, ${ }^{4}$ \\ Soe Mar, MD, ${ }^{5}$ Michelle M. Miller-Thomas, MD, ${ }^{2}$ Joshua S. Shimony, MD, PhD, ${ }^{2}$ \\ Matthew D. Smyth, MD, ${ }^{4}$ and Tammie L. S. Benzinger, MD, PhD ${ }^{2}$
}

\begin{abstract}
${ }^{1}$ Department of Medical Imaging, Neuroradiology Section, University of Arizona, Tucson, Arizona; ${ }^{2}$ Neuroradiology Section, Mallinckrodt Institute of Radiology, Washington University; ${ }^{3}$ Department of Pathology and Immunology, Washington University; ${ }^{4}$ Department of Neurosurgery, Pediatric Division, St. Louis Children's Hospital/Washington University; and 5Department of Neurology, Division of Pediatric Neurology, Washington University School of Medicine, St. Louis, Missouri
\end{abstract}

OBJECTIVE Many patients with medically intractable epilepsy have mesial temporal sclerosis (MTS), which significantly affects their quality of life. The surgical excision of MTS lesions can result in marked improvement or even complete resolution of the epileptic episodes. Reliable radiological diagnosis of MTS is a clinical challenge. The purpose of this study was to evaluate the utility of volumetric mapping of the hippocampi for the identification of MTS in a case-controlled series of pediatric patients who underwent resection for medically refractory epilepsy, using pathology as a gold standard.

METHODS A cohort of 57 pediatric patients who underwent resection for medically intractable epilepsy between 2005 and 2015 was evaluated. On pathological investigation, this group included 24 patients with MTS and 33 patients with non-MTS findings. Retrospective quantitative volumetric measurements of the hippocampi were acquired for 37 of these 57 patients. Two neuroradiologists with more than 10 years of experience who were blinded to the patients' MTS status performed the retrospective review of MR images. To produce the volumetric data, MR scans were parcellated and segmented using the FreeSurfer software suite. Hippocampal regions of interest were compared against an age-weighted local regression curve generated with data from the pediatric normal cohort. Standard deviations and percentiles of specific subjects were calculated. The sensitivity, specificity, positive predictive value (PPV), and negative predictive value (NPV) were determined for the original clinical read and the expert readers. Receiver operating characteristic curves were generated for the methods of classification to compare results from the readers with the authors' results, and an optimal threshold was determined. From that threshold the sensitivity, specificity, PPV, and NPV were calculated for the volumetric analysis.

RESULTS With the use of quantitative volumetry, a sensitivity of $72 \%$, a specificity of $95 \%$, a PPV of $93 \%$, an NPV of $78 \%$, and an area under the curve of 0.84 were obtained using a percentage difference of normalized hippocampal volume. The resulting specificity (95\%) and PPV (93\%) are superior to the original clinical read and to Reader A and Reader B's findings (range for specificity $74 \%-86 \%$ and for PPV $64 \%-71 \%)$. The sensitivity (72\%) and NPV (78\%) are comparable to Reader A's findings (73\% and $81 \%$, respectively) and are better than those of the original clinical read and of Reader B (sensitivity $45 \%$ and $63 \%$ and NPV $71 \%$ and $70 \%$, respectively).

CONCLUSIONS Volumetric measurement of the hippocampi outperforms expert readers in specificity and PPV, and it demonstrates comparable to superior sensitivity and NPV. Volumetric measurements can complement anatomical imaging for the identification of MTS, much like a computer-aided detection tool would. The implementation of this approach in the daily clinical workflow could significantly improve diagnostic accuracy.

https://thejns.org/doi/abs/10.3171/2016.12.PEDS16335

KEY WORDS advanced imaging techniques; mesial temporal sclerosis; magnetic resonance imaging; epilepsy surgery

ABBREVIATIONS AUC = area under the curve; LOWESS = locally weighted scatterplot smoothing; MPRAGE = magnetization-prepared rapid acquisition gradient echo; MTS = mesial temporal sclerosis; NPV = negative predictive value; PNC = pediatric normal cohort; PPV = positive predictive value; $\mathrm{QC}=$ quality control.

SUBMITTED June 7, 2016. ACCEPTED December 8, 2016.

INCLUDE WHEN CITING Published online March 24, 2017; DOI: 10.3171/2016.12.PEDS16335. 
$\mathrm{M}$ ESIAL temporal sclerosis (MTS) of the hippocampus is a known cause of intractable epilepsy in the pediatric population. However, its true incidence and pathogenesis are not known. Pathological evaluation shows neuronal loss, gliosis of the hippocampus, and axonal reorganization. ${ }^{24}$ These features are associated with epileptogenic foci that may be isolated or that may occur together with other cortical malformations (i.e., dual pathology).,4 The surgical excision of MTS lesions can result in marked improvement, if not complete resolution, of the epileptic episodes. However, reliable radiological diagnosis of MTS is a clinical challenge.

In conjunction with a battery of clinical and electrophysiological studies, MRI is routinely used during the preoperative evaluation of children with epilepsy. ${ }^{3,13}$ Widely accepted MRI features of MTS include diminished hippocampal volume, increased hippocampal signal intensity on T2-weighted and FLAIR sequences, decreased temporal lobe size, blurring of the gray matter-white matter differentiation in the temporal lobe, atrophy of the adjacent hippocampal collateral white matter, and an enlarged ipsilateral temporal horn. Because MTS is widely recognized as a progressive disease, hippocampal volume decrease may not be as evident in the pediatric population as it is in adult patients. ${ }^{12}$

In the present study, we used a case-control cohort of 57 pediatric patients to investigate whether the volumetric quantitative assessment of hippocampal volume with newer paradigms based on the FreeSurfer software suite improved the diagnostic accuracy of MTS in presurgical patients as compared with the qualitative evaluation of anatomical images only. The prognostic value was analyzed for the sensitivity, specificity, positive predictive value (PPV), and negative predictive value (NPV) of this method for the diagnosis of MTS as compared with the traditional anatomical morphological findings that are typically used for its radiological diagnosis. Our aims were to improve the accuracy of MTS diagnosis in the pediatric population and to improve the prognostic value of imaging methods to ensure better postoperative outcomes for these patients.

\section{Methods}

\section{Pediatric Normal Cohort Definition and Use}

A pediatric normal cohort (PNC) of 89 patients between the ages of 2 and 19 years with normal results on brain MR images was identified retrospectively. The PNC was used to correct for changes in brain size and growth according to age. The PNC members were identified on the basis of their referral for clinical brain MRI, with the most common indication being headache; their initial examination interpretations were normal, there was clinical confirmation of no seizure disorder, and no developmental delays or syndromic diseases were present. The MR images of these patients were then visually confirmed as normal by a second neuroradiologist (G.J.G.P.C. or T.L.S.B.). We used 1.5-T and 3-T sagittal magnetization-prepared rapid acquisition gradient echo (MPRAGE) sequences for FreeSurfer normalized data generation. No patients were excluded due to pathology. However, 21 cases could not be used due to motion artifact $(n=5)$; poor gray matter-white matter con- trast on the T1-weighted image, atlas registration failure, or both $(n=1)$; or incorrect T1 protocol $(n=15)$. For the analyses, the 68 PNC scans were grouped by age: either 2 to 12 years old ( $n=43$; mean age 8.0 years; SD 2.6 years) or 9 to 19 years old $(n=43$; mean age 13.3 years; SD 3.1 years). The purpose of the age overlap was to make the groups even, and to allow the weighted regression to handle the edges of the age range better. Scans were a mixture of $1.5 \mathrm{~T}(\mathrm{n}=52)$ and $3 \mathrm{~T}(\mathrm{n}=37)$ for the cohort as a whole. When divided into the age ranges, the groups included 21 (49\%) 3-T scans within the 43 images selected for the 2to 12-year-old group and 17 (39\%) 3-T scans from those obtained in the 43 patients in the 9- to 19-year-old group.

The lack of volumetric information beyond the age range given causes the standard error of the locally weighted scatterplot smoothing (LOWESS) regression to grow larger toward the ends of the age ranges regressed, thus making any estimation for standard deviation larger as well. To correct for this, ages were chosen outside the desired range of ages for testing, which were 2-10 years and 11-19 years, and the ranges used in the LOWESS regression were 2-12 years and 9-19 years. Although the ranges were expanded to account for problems with standard error at the ends of a range, only individuals 2-10 years and 11-19 years of age were tested in their respective individual longitudinal participant tests. Unfortunately, no controls were found for ages above 19 years and below 2 years to address standard error issues in those areas.

All FreeSurfer data generated for the PNC, the study group, and the control group were created using the standard FreeSurfer atlas with FreeSurfer version 6.0-beta.

We normalized both the left and right hippocampal volumes of the PNC to the PNC's intracranial volumes by using the following formula: Vnorm = Vorig $-B(I C V$ - ICVave), where Vnorm is the normalized volume after adjustments; Vorig is the original volume to be normalized; $B$ is the unstandardized regression coefficient from the linear regression between the hippocampal region and the intracranial volume of the PNC; ICV is the intracranial volume of the scan to be normalized; and ICVave is the average intracranial volume within the PNC. After the PNC volumes were normalized, an age-weighted local regression ${ }^{5}$ between each patient's hippocampal volume and his or her age at the time was created, with a degree of 1 and a span of 0.7. An average line-along with the line's standard error-was generated using the "predict" function from the $\mathrm{R}$ language's statistics package, with which the local regression could be plotted and the standard deviation calculated. ${ }^{14}$ The average line predicted from the local regression represented what approximates a natural atrophy or growth line due to aging. The z-score range for the local regression was not calculated as a function of the whole weighted regression; rather, the z-score was calculated for each integer age between 2 and 10 years and shown with a shaded area and a dotted gray line. The same steps were taken for graphs that depicted the ages between 10 and 19 years.

Each patient had his or her own hippocampal volume normalized in the same way as for the PNC, and his or her normalized results were displayed on a graph, as shown in Fig. 1. 


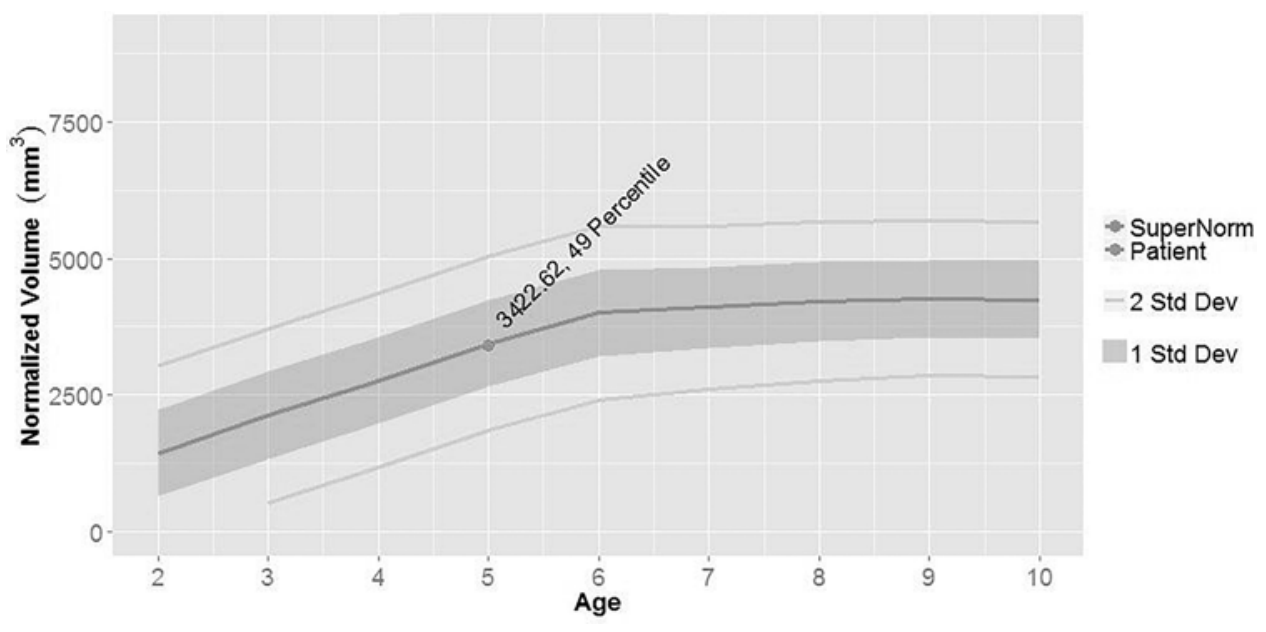

FIG. 1. Example of hippocampal volumetric quantitative graph, expressed as an age-normalized percentile.

\section{Characteristics of Patients With Epilepsy}

After obtaining institutional review board approval, the MR images, clinical pathology data, and clinical outcome data (Engel class) $)^{9}$ were obtained in 57 patients who had undergone resection for medically intractable epilepsy between 2005 and 2015. The areas under the curve (AUCs) and the thresholds used to generate the statistics are shown in Table 1. Only patients with a nonischemic and nontumoral histologically proven diagnosis were included. Patients with dual pathology were excluded. Pathological investigation proved MTS in 24 patients, and 33 had other histologically proven pathologies (Tables 2 and 3).

Of these 57 patients, 20 were excluded for various reasons, such as incomplete or unavailable preoperative MRI studies, no MPRAGE available for FreeSurfer evaluation, large areas of tissue loss, poor gray matter-white matter differentiation due to patient age ( $<6$ months), and technical failure that precluded FreeSurfer evaluation. These cases are detailed in Table 3.

After mesial temporal resection, seizure outcomes were determined in patients according to Engel class by a neurosurgery resident (A.K.V.) through evaluation of the last postoperative follow-up neurosurgical clinical note.

\section{Evaluation of MRI Studies}

The MRI studies were performed only on a 3-T Magnetom Trio scanner (Siemens Medical Solutions, Erlangen, Germany). The epilepsy patients $(n=57)$ underwent the following standard clinical epilepsy protocol: 1) sagittal
MPRAGE with 1-mm isotropic voxels; 2) axial FLAIR, with a slice thickness of $5 \mathrm{~mm}$; 3) coronal FLAIR, with a slice thickness of $3 \mathrm{~mm}$; 4) coronal T2-weighted turbo spin echo, with a slice thickness of $3 \mathrm{~mm} ; 5$ ) axial T2-weighted turbo spin echo, with a slice thickness of $5 \mathrm{~mm}$; and 6) axial diffusion-weighted imaging/apparent diffusion coefficient, with 25 diffusion directions, $b$ values of 0 and 1000 seconds $/ \mathrm{mm}^{2}$, and a slice thickness of $5 \mathrm{~mm}$. Patients in the PNC group $(n=89)$ underwent standard clinical brain MRI with no dedicated seizure protocol sequences (MPRAGE, coronal FLAIR, and coronal T2-weighted turbo spin echo), because they presented at the emergency department or outpatient clinic with complaints of headache.

\section{Preoperative Evaluation of MRI}

The original radiological reports for preoperative MRI were reviewed and labeled as the clinical read. All the sequences of the preoperative MRI studies were evaluated by board-certified faculty of a tertiary academic neuroradiology department, all with more than 10 years of experience.

\section{Retrospective Evaluation of MRI}

As previously described, ${ }^{15} 2$ board-certified neuroradiologists (J.S.S. and M.M.M.T.), who were blinded to patient conditions, reviewed only the MPRAGE, T2-weighted, and FLAIR sequences of all epilepsy patients for the 6 MTS features described by Meiners and colleagues: $:^{18} 1$ ) increased hippocampal signal intensity; 2) reduced hippocampal size; 3) atrophy of the ipsilateral hippocampal col-

TABLE 1. Statistics generated using the volumetric data in 57 patients with intractable epilepsy

\begin{tabular}{lcccccc}
\hline Parameter & Clinical Read & Reader A & Reader B & z-Score Diff & Normalized Vol Diff & Normalized Volumetric \% Diff \\
\hline Threshold & NA & NA & NA & 0.13 & 658 & 0.17 \\
\hline AUC & NA & NA & NA & 0.68 & 0.78 & 0.84 \\
\hline Sensitivity & 0.45 & 0.73 & 0.63 & 0.94 & 0.61 & 0.72 \\
\hline Specificity & 0.86 & 0.74 & 0.77 & 0.42 & 0.95 & 0.95 \\
\hline PPV & 0.67 & 0.64 & 0.71 & 0.60 & 0.92 & 0.93 \\
\hline NPV & 0.71 & 0.81 & 0.70 & 0.88 & 0.72 & 0.78 \\
\hline
\end{tabular}

Diff = difference; $N A=$ not applicable. 
TABLE 2. Summary of clinical and histopathological characteristics of 37 pediatric patients with intractable epilepsy whose data were processed with FreeSurfer to generate volumetrics

\begin{tabular}{|c|c|c|c|c|c|c|c|c|c|c|c|c|}
\hline \multirow{2}{*}{$\begin{array}{l}\text { Case } \\
\text { No. }\end{array}$} & \multicolumn{2}{|c|}{ Hippocampal Vol } & \multicolumn{3}{|c|}{ z-Score } & \multirow{2}{*}{$\begin{array}{l}\text { Normalized } \\
\text { Vol Diff }^{\star}\end{array}$} & \multirow{2}{*}{$\begin{array}{l}\text { Volumetric } \\
\% \text { Diff }\end{array}$} & \multirow{2}{*}{$\begin{array}{l}\text { Age at } \\
\text { Op (mos) }\end{array}$} & \multirow[b]{2}{*}{ Preop MRI Findings } & \multirow{2}{*}{$\begin{array}{l}\text { Side } \\
\text { of Op }\end{array}$} & \multirow[b]{2}{*}{ MTS } & \multirow{2}{*}{$\begin{array}{l}\text { Engel Class } \\
\text { at Last FU }\end{array}$} \\
\hline & $\mathrm{Lt}$ & Rt & $\mathrm{Lt}$ & Rt & Diff* $^{\star}$ & & & & & & & \\
\hline 3 & 4113.693 & 4274.301 & 0.337 & 0.484 & -0.147 & -160.608 & -0.038 & 197 & Normal & Rt & Yes & ID \\
\hline 5 & 4068.107 & 4498.037 & 0.984 & 1.553 & -0.569 & -429.93 & -0.1 & 61 & MTS & Lt & No & IA \\
\hline 9 & 3777.382 & 4134.868 & -0.201 & 0.197 & -0.398 & -357.486 & -0.09 & 187 & Other & Lt & No & IA \\
\hline 11 & 4146.788 & 4253.204 & 0.471 & 0.488 & -0.017 & -106.416 & -0.025 & 241 & Normal & Rt & No & IVB \\
\hline 12 & 4436.118 & 4289.424 & 1.36 & 1.366 & -0.006 & 146.695 & 0.034 & 50 & Other & $\mathrm{Lt}$ & No & IA \\
\hline 13 & 4895.581 & 5195.92 & 0.817 & 0.763 & 0.055 & -300.339 & -0.06 & 143 & Normal & Rt & No & IC \\
\hline 14 & 3844.653 & 4501.972 & 0.068 & 0.642 & -0.575 & -657.319 & -0.158 & 196 & Sequela of infarct & $\mathrm{Rt}$ & No & Lost to FU \\
\hline 15 & 4311.439 & 3422.622 & 0.87 & -0.027 & 0.897 & 888.817 & 0.23 & 62 & Normal & Rt & Yes & IC \\
\hline 17 & 2882.888 & 2670.961 & -2.009 & -2.118 & 0.109 & 211.926 & 0.076 & 104 & Normal & $\mathrm{Rt}$ & Yes & IA \\
\hline 21 & 3454.156 & 3341.866 & -0.243 & -0.119 & -0.124 & 112.29 & 0.033 & 48 & Chiari I & $\mathrm{Lt}$ & No & IIIA \\
\hline 24 & 3339.21 & 3764.575 & -0.629 & -0.053 & -0.576 & -425.365 & -0.12 & 185 & MTS & Lt & Yes & IA \\
\hline 25 & 4460.404 & 4663.704 & 0.271 & 0.265 & 0.005 & -203.3 & -0.045 & 4 & FCD & $\mathrm{Lt}$ & No & IA \\
\hline 27 & 3113.933 & 2626.666 & -0.863 & -0.876 & 0.013 & 487.267 & 0.17 & 149 & Other & $\mathrm{Rt}$ & No & IA \\
\hline 28 & 4072.057 & 2717.468 & 0.399 & -0.357 & 0.757 & 1354.589 & 0.399 & 216 & MTS & $\mathrm{Rt}$ & Yes & IA \\
\hline 29 & 3557.217 & 4377.225 & -1.005 & 0.155 & -1.16 & -820.008 & -0.207 & 108 & Parietal t & Lt & Yes & IVA \\
\hline 31 & 4280.172 & 3512.733 & 0.504 & -0.043 & 0.548 & 767.439 & 0.197 & 193 & MTS & Rt & Yes & $\| \mathrm{A}$ \\
\hline 32 & 4377.961 & 3889.004 & 0.654 & 0.316 & 0.338 & 488.957 & 0.118 & 210 & Normal & Lt & No & IA \\
\hline 33 & 2564.68 & 4448.995 & -2.375 & 0.464 & -2.839 & -1884.315 & -0.537 & 90 & MTS & Lt & Yes & IA \\
\hline 34 & 4674.7 & 4223.578 & 0.81 & 0.024 & 0.786 & 451.122 & 0.101 & 106 & $\begin{array}{l}\text { Encephalomalacia \& } \\
\text { abnormal WM signal }\end{array}$ & $\mathrm{Rt}$ & No & IVB \\
\hline 35 & 4398.464 & 4336.479 & 0.301 & 0.146 & 0.155 & 61.985 & 0.014 & 17 & $\mathrm{FCD}$ & $\mathrm{Rt}$ & No & IA \\
\hline 36 & 3027.03 & 2543.03 & -0.806 & -1.036 & 0.23 & 484.00 & 0.174 & 73 & MTS & $\mathrm{Rt}$ & Yes & IVC \\
\hline 39 & 2971.526 & 2207.293 & -1.129 & -1.274 & 0.144 & 764.233 & 0.295 & 184 & Normal & Lt & Yes & IIIA \\
\hline 40 & 3637.076 & 5190.563 & -0.755 & 1.235 & -1.99 & -1553.486 & -0.352 & 126 & MTS & Lt & No & IIIA \\
\hline 41 & 3433.632 & 4389.717 & -0.132 & 0.604 & -0.736 & -956.085 & -0.244 & 204 & MTS & Lt & Yes & IA \\
\hline 42 & 3470.645 & 2524.235 & 0.88 & 0.354 & 0.526 & 946.41 & 0.316 & 40 & MTS & $\mathrm{Rt}$ & Yes & IA \\
\hline 43 & 3466.416 & 3460.963 & -0.99 & -0.866 & -0.124 & 5.453 & 0.002 & 107 & Normal & $\mathrm{Rt}$ & No & IB \\
\hline 45 & 4385.789 & 4022.57 & 0.333 & -0.347 & 0.679 & 363.219 & 0.086 & 109 & Normal & $\mathrm{Lt}$ & No & IA \\
\hline 46 & 3908.544 & 3994.615 & 0.263 & 0.377 & -0.113 & -86.071 & -0.022 & 206 & FCD & Lt & No & IVC \\
\hline 47 & 3259.616 & 3196.103 & 0.158 & 1.571 & -1.413 & 63.513 & 0.02 & 27 & FCD & Lt & No & IA \\
\hline 48 & 3120.495 & 2905.466 & 0.015 & 1.311 & -1.295 & 215.029 & 0.071 & 20 & $\mathrm{FCD}$ & $\mathrm{Rt}$ & No & IA \\
\hline 49 & 3449.572 & 3503.825 & -0.328 & -0.05 & -0.279 & -54.253 & -0.016 & 199 & MTS & $\mathrm{Rt}$ & Yes & IA \\
\hline 51 & 2298.638 & 1847.82 & -0.688 & -0.535 & -0.152 & 450.818 & 0.217 & 222 & Normal & $\mathrm{Lt}$ & Yes & IA \\
\hline 52 & 2897.364 & 2030.084 & -1.061 & -1.225 & 0.164 & 867.281 & 0.352 & 146 & MTS & $\mathrm{Rt}$ & Yes & Lost $t$ \\
\hline 54 & 4102.455 & 3300.422 & 0.449 & 0.169 & 0.281 & 802.033 & 0.217 & 227 & MTS & $\mathrm{Rt}$ & Yes & IA \\
\hline 55 & 4426.789 & 4740.188 & 0.651 & 0.807 & -0.156 & -313.398 & -0.068 & 210 & Normal & Lt & No & IB \\
\hline 56 & 3870.645 & 4066.392 & -0.369 & -0.051 & -0.318 & -195.747 & -0.049 & 88 & MTS & Lt & Yes & IA \\
\hline 57 & 2327.147 & 3072.558 & -1.618 & -0.521 & -1.097 & -745.411 & -0.276 & 180 & MTS & Lt & Yes & IA \\
\hline
\end{tabular}

$\mathrm{FCD}=$ focal cortical dysplasia; $\mathrm{FU}=$ follow-up; $\mathrm{WM}=$ white matter.

* Differences were calculated as left minus right.

lateral white matter; 4) enlarged ipsilateral temporal horn; $5)$ reduced gray matter-white matter demarcation in the temporal lobe; and 6) decreased temporal lobe size. They then classified the results as left MTS, right MTS, nonMTS, or normal. The non-MTS classification included all pathological findings that were not compatible with MTS, such as tumor, vascular malformations, and so on. Scans classified as normal had no pathological findings. The ini- tial preoperative findings from the clinical read were used in the statistical analysis, together with the findings from Readers A and B, who performed similar services for the original article by Kasasbeh and colleagues. ${ }^{15}$

\section{Volumetric Hippocampal Volume Acquisition}

To produce the volumetric data, the T1-weighted MPRAGE images were parcellated and segmented with 
TABLE 3. Summary of clinical and histopathological characteristics of 20 pediatric patients with intractable epilepsy whose data could not be processed with FreeSurfer to generate volumetrics

\begin{tabular}{|c|c|c|c|c|c|c|}
\hline $\begin{array}{l}\text { Case } \\
\text { No. }\end{array}$ & Cause of FreeSurfer Failure or Reason for Exclusion & $\begin{array}{c}\text { Age at } \\
\text { Op (mos) }\end{array}$ & $\begin{array}{l}\text { Preop MRI } \\
\text { Findings }\end{array}$ & $\begin{array}{l}\text { Side } \\
\text { of Op }\end{array}$ & MTS & $\begin{array}{l}\text { Engel Class } \\
\text { at Last FU }\end{array}$ \\
\hline 1 & No preop MRI available & 23 & Atrophic It hemisphere & Lt & Yes & IA \\
\hline 2 & No preop MRI available & 164 & Atrophic It hemisphere & Lt & Yes & IA \\
\hline 4 & No preop MRI available & 187 & Normal & Lt & Yes & IA \\
\hline 6 & No preop MRI available & 159 & Normal & Lt & No & $\| A$ \\
\hline 7 & Encephalomalacia \& an infarct, registration failure & 61 & Other & Rt & No & IC \\
\hline 8 & No preop MRI available & 47 & Normal & Lt & No & IA \\
\hline 10 & No preop MRI available & 60 & Normal & Rt & No & $\| A$ \\
\hline 16 & Patient too young (<6 mos old) for valid comparison w/ normal group & 5 & Other & Rt & No & IIIA \\
\hline 18 & Encephalomalacia, registration failure & 101 & Other & Lt & Yes & IA \\
\hline 19 & No precontrast volumetric T1-weighted images; patient also had heterotopia & 33 & Other & Rt & No & IA \\
\hline 20 & Encephalomalacia, infarction, gliosis, \& WM atrophy; registration failure & 246 & Other & Lt & No & IA \\
\hline 22 & No precontrast volumetric T1-weighted images & 194 & Other & $\mathrm{Lt}$ & No & IA \\
\hline 23 & Encephalomalacia, registration failure & 192 & Other & Rt & No & IA \\
\hline 26 & Patient too young (<6 mos old) for valid comparison w/ normal group & 16 & Other & Lt & No & $\| A$ \\
\hline 30 & Patient had encephalomalacia & 201 & Other & $\mathrm{Rt}$ & No & IIB \\
\hline 37 & Patient too young (<6 mos old) for valid comparison w/ normal group & 6 & Normal & Lt & No & NA \\
\hline 38 & Encephalomalacia from tumor resection, registration failure & 142 & Other & Rt & No & IA \\
\hline 44 & Talairach failure due to poor WM-GM contrast & 90 & MTS & $\mathrm{Rt}$ & Yes & IA \\
\hline 50 & No precontrast volumetric T1-weighted images & 194 & Choroidal cyst/DNET & Rt & No & IA \\
\hline 53 & No preop MRI available & 19 & Postop changes & $\mathrm{Rt}$ & Yes & IVB \\
\hline
\end{tabular}

DNET = dysembryoplastic neuroepithelial tumor; $\mathrm{GM}$ = gray matter.

FreeSurfer, which is an automated segmentation and parcellation tool developed by the Laboratory of Computational Imaging at Massachusetts General Hospital (Martinos, Massachusetts General Hospital, Boston, MA). FreeSurfer has been used in more than 200 publications for a wide variety of neuroimaging topics. A full list of cited documents can be found at the website: www.zotero. org/freesurfer.

The majority of work performed using FreeSurfer involves assessments in adults; however, the use of FreeSurfer in children has been validated in a 2010 paper by Ghosh et al. ${ }^{11}$ In the aforementioned paper, 31 children between the ages of 4 and 11 years underwent scanning and processing both manually and via FreeSurfer. The results show that spatial normalization to a common reference frame (via FreeSurfer) produces accurate results without introducing an age-related bias, and FreeSurfer processes were comparable to, if not better than, a diffeomorphic-registration algorithm. We rely on Ghosh's validation of FreeSurfer use in children, and in this paper we used FreeSurfer to find accurate volumetrics for use in predictive measurements.

Some of the patient data were not isometric, which FreeSurfer requires. Thus, nonisometric scans had to be resampled to isometric space. To ensure that all of the scans were processed the same way, all other electronic MRI files (DICOM) were run through the same resampling software, even if they were isometric. Quality control (QC) was performed by an expert in FreeSurfer requirements (C.O.). The QC protocol entails comparing FreeSurfer surface calculations and the QC rater's view of the gray matter-white matter boundary and of the pial boundary. If FreeSurfer's boundaries were questionable and the QC rater was certain of incorrect boundary and label generation, then corrections were run in accordance with tutorials for editing on the Freesurfer Wiki. Note that rerunning edits requires anywhere from just a few hours to a half day of reprocessing. If FreeSurfer's boundaries are questionable but the QC rater is uncertain whether boundaries were generated correctly, FreeSurfer is left unedited.

Becoming proficient in performing QC can take approximately 1 week, at which point performing QC for each FreeSurfer result will take anywhere from 10 to 30 minutes. When someone becomes more experienced with FreeSurfer QC, the process of performing QC can be shortened to 5-10 minutes for pediatric patients.

Individual regions of interest were calculated and normalized via a comparison of each patient's intracranial volume to the intracranial volume of a PNC member. ${ }^{10}$ After normalization, the regions of interest were compared with an age-weighted local regression curve generated with the use of the PNC. ${ }^{5}$ Standard deviations and percentiles of specific subjects were calculated from the aforementioned local regression.

\section{Results}

\section{Patient Characteristics}

Tables 2 and 3 summarize patient demographic data, clinical profiles, histopathological results, Engel class at last follow-up, and preoperative MRI diagnoses. In this 
study, $12 \%(7 / 57)$ of studies could not be processed due to a lack of preoperative imaging; 5\% (3/57) due to a lack of precontrast MPRAGE images; 11\% (6/57) due to large areas of tissue loss from encephalomalacia, infarct, or postoperative changes; and 5\% (3/57) due to poor gray matterwhite matter differentiation (patient $<6$ months old). In addition, 2\% (1/57) could not be included due to technical failure that precluded FreeSurfer evaluation (see Table 3).

\section{Statistical Analysis}

Three methods for categorization were used: 1) the difference between the normalized hippocampal volume per side; 2) the percentage difference between the normalized hippocampal volume per side; and 3) the difference between the $\mathrm{z}$-scores for each side. The absolute value of each classification was used, because the tests used to evaluate whether MTS is present are based on a difference in hippocampal volume. To show which side was in fact larger in each patient, the positive and negative values are shown in Table 2. Each method for automatic classification was tested with the use of receiver operating characteristic curves to determine the best threshold to use. These curves were evaluated by the best AUC. The AUCs and the thresholds used to generate the statistics are shown in Table 1. Specifically, the calculated AUCs were 0.68 for z-score difference, 0.78 for normalized volume difference, and 0.84 for volumetric percent difference (Fig. 2).

\section{Anatomical Evaluation of Clinical Read, Reader A, and Reader B}

With the use of FreeSurfer-based volumetry, a sensitivity of $72 \%$, a specificity of $95 \%$, a PPV of $93 \%$, an NPV of $78 \%$, and an AUC of 0.84 were obtained using a percentage difference of normalized hippocampal volume. Clinical read, Reader A, and Reader B had differences in these areas: sensitivity, 45\%-73\%; specificity, 74\%-86\%; NPV, 70\%-81\%; and PPV, 64\%-71\%. Table 1 provides these data, and it details both the FreeSurfer volumetry statistics and the recalculated statistics for clinical read, Reader A, and Reader B for all 57 individuals within the case-control group.

\section{Discussion}

Mesial temporal sclerosis is a known cause of intractable epilepsy in the pediatric population. It is a condition that affects the hippocampus, although it is not uniformly affected, with the dentate gyrus and the CA1, CA4, and CA3 (to a lesser degree) sections of the hippocampus being primarily involved. ${ }^{4}$ Histologically, there is neuronal cell loss, gliosis, and sclerosis in the hippocampus (Fig. 3). The sclerotic hippocampus is distinguished from the normal hippocampus by neuronal loss and gliosis, particularly in the CA1 subfield and the end folium of the dentate hilum. ${ }^{4}$

The estimated lifetime prevalence of epilepsy or seizure disorder is 10.2 per 1000 individuals, and the prevalence of current epilepsy or seizure disorder is 6.3 per $1000 .^{22}$ The exact incidence of MTS is unknown and difficult to determine, ${ }^{23}$ although the prevalence has been reported as $3.1 \%$ in all brain MRI overall and as $12.1 \%$ in all brain MRI examinations performed to evaluate for seizures. ${ }^{20}$

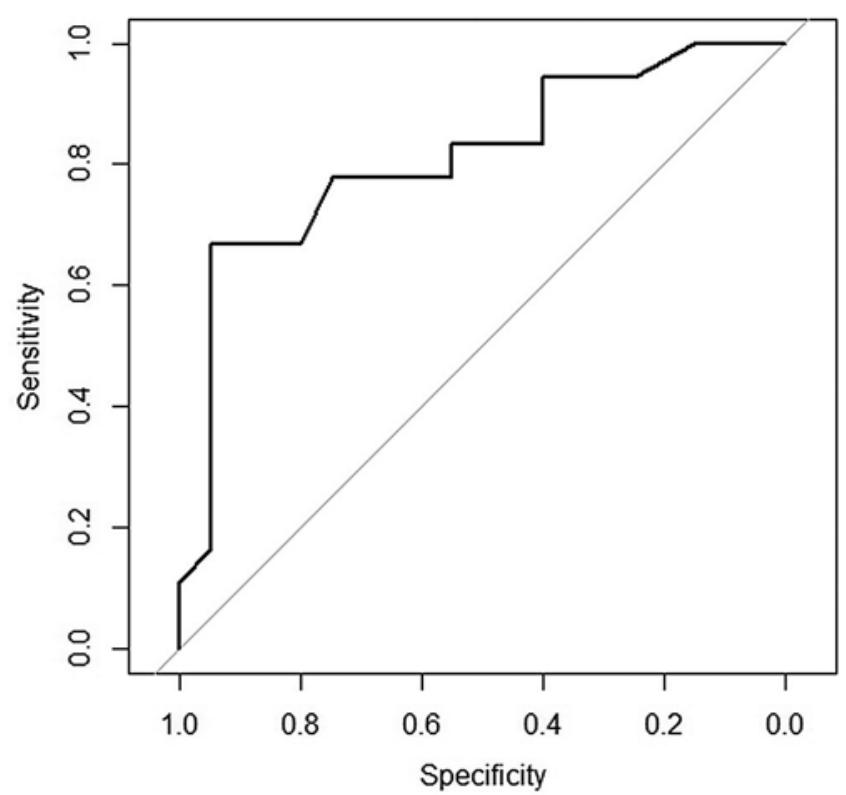

FIG. 2. Graph showing the AUC of the raw volumetric percent difference.

As well, Ng et al. described a $0.77 \%$ rate of MTS in children undergoing MRI scans, of whom all with MTS had seizures. ${ }^{19}$ In appropriate surgical candidates, there is an approximately $80 \%$ chance of being seizure free at 1 year postoperatively, with outcomes being influenced by several factors, including preoperative hippocampal sclerosis and seizure localized to the anterior temporal lobe. ${ }^{17}$ Because MTS is a known cause of intractable epilepsy in the pediatric population, the appropriate identification of these patients is essential to ensure a favorable postsurgical outcome.

Since 1995, 6 articles evaluating hippocampal volumetry for the diagnosis of MTS have been published, according to a PubMed search conducted in March 2016.,16-8, ${ }_{16,21}$ To our knowledge, only the present study evaluates pediatric patients with MTS, and we have also provided clinical profiles, preoperative MRI diagnoses, histopathological results, and Engel class. Our study is also the only one that compares the performance of FreeSurfer software and expert neuroradiology readers.

In our study, with the use of hippocampal volumetry, a sensitivity of $72 \%$, a specificity of $95 \%$, a PPV of $93 \%$, an NPV of $78 \%$, and an AUC of 0.84 were obtained using a percentage difference of normalized hippocampal volume. The resulting specificity (95\%) and PPV (93\%) are superior to those obtained from the original clinical read and Readers A and B (74\%-86\% and 64\%-71\%, respectively). The sensitivity (72\%) and NPV (78\%) are comparable to Reader A's findings (73\% and $81 \%$, respectively) and better than those of the original clinical read and Reader B (sensitivity $45 \%$ and $63 \%$ and NPV $71 \%$ and $70 \%$, respectively). Please see Table 1 for details.

For example, Case 15 was read as normal by all readers. It was eventually diagnosed as histologically proven right MTS, and it was only correctly identified by quantitative analysis (Fig. 4). Alternatively, Case 24 was correctly identified by all readers as left MTS by using only anatomical 

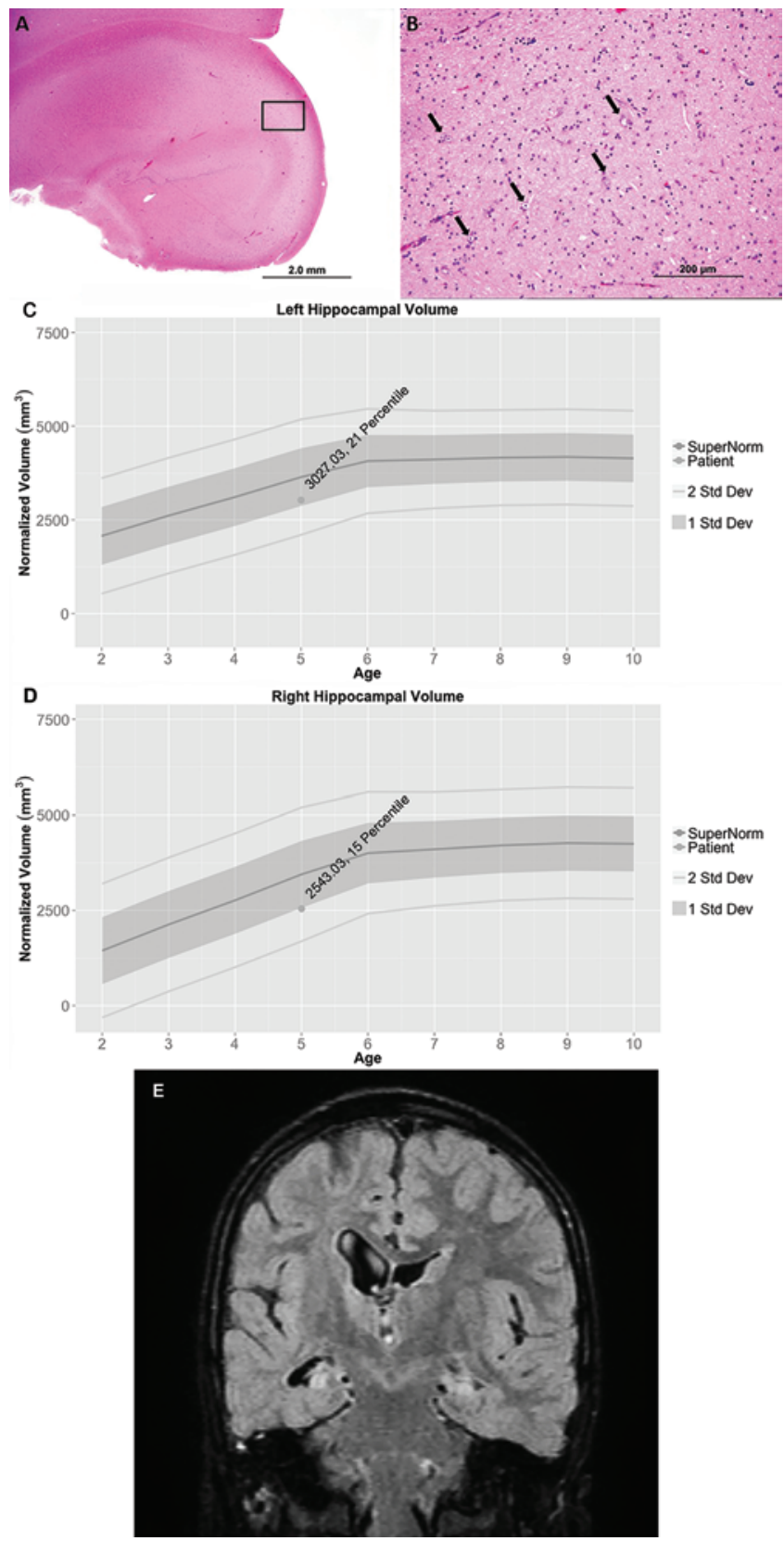

FIG. 3. Case 36. A: A low-power photomicrograph of the hippocampus in the region of the dentate gyrus. $\mathrm{H} \& \mathrm{E}$, original magnification $\times 20$. B: A higher-power photomicrograph of the outlined region in panel A showing the CA1 region, with marked neuronal loss and gliosis; arrows indicate the few remaining neurons. $\mathrm{H} \& \mathrm{E}$, original magnification $\times 200$. C: Corresponding left hippocampal volumetric quantitative graph. D: Corresponding right hippocampal volumetric quantitative graph. E: Corresponding coronal FLAIR image showing the right MTS. This patient's original MRI clinical report and Readers A and B correctly identified the patient's pathology. Figure is available in color online only.

imaging, and it was also was correctly identified by FreeSurfer (Fig. 5).

Interestingly, 3 cases $(3,39$, and 49$)$ demonstrated slightly increased hippocampal volume on the affected side (Fig. 6). This may be found during very early disease, with ac-
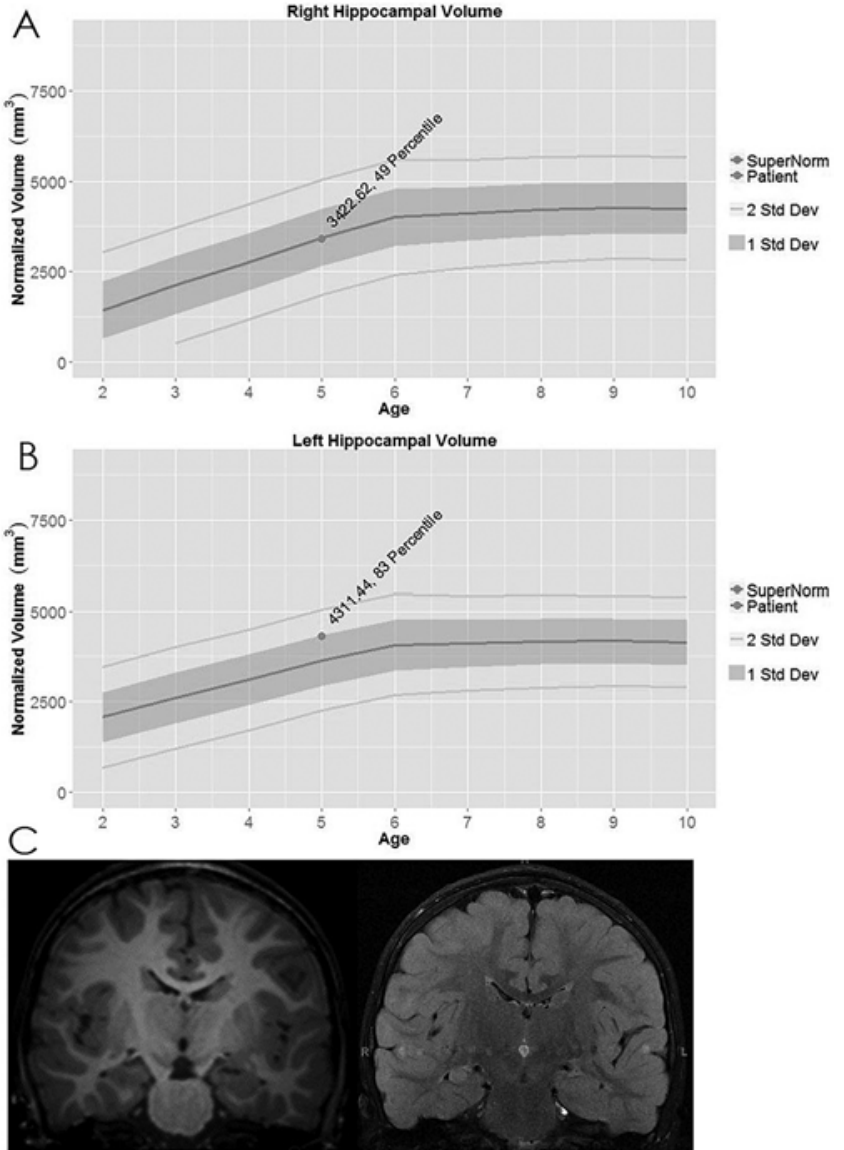

FIG. 4. Case 15. Right MTS proven on pathological investigation. Note that in panel $\mathbf{A}$, the right hippocampal volume is less than in panel $\mathbf{B}$, which shows the left hippocampal volume. This patient's original clinical $M R I$ read $(\mathbf{C})$ was incorrectly described as normal, but correctly identified as MTS by Reader A and B.

companying inflammatory changes that may transiently increase tissue volume before atrophy develops. ${ }^{25}$

It is notable that quantitative hippocampal volumetry was better at identifying MTS than a cohort of board-certified academic neuroradiologists with more than 10 years' experience each. This indicated that a system akin to the computer-aided detection software used in mammography may aid even an expert neuroradiologist to better recognize these lesions.

Seizure outcome at follow-up as measured by the Engel class was not found to have a statistically significant association with the correct preoperative identification of MTS. Because this research was retrospective, it had no impact on the surgeon's treatment choices. These findings mirror those previously reported..$^{15}$

A significant number of cases (20 of 57 [35\%]) could not be processed due to a combination of factors. These factors include a lack of preoperative or precontrast volumetric T1-weighted sequences, which is required. Other contributing factors include large areas of tissue loss due to encephalomalacia, infarct, or postoperative changes that likewise interfere with FreeSurfer's correct normalization of data. There was a single registration failure due to poor 

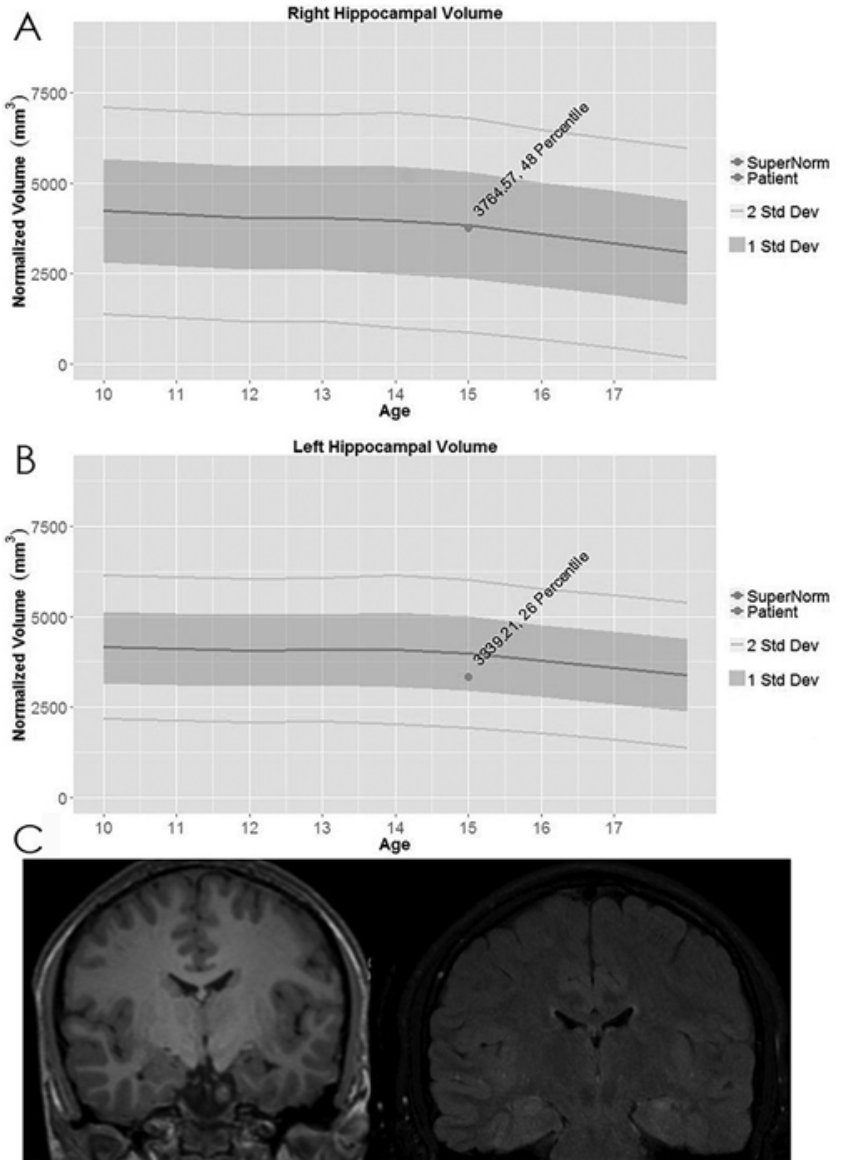

FIG. 5. Case 24. Left MTS proven on pathological investigation. Note that in panel $\mathbf{B}$, the left hippocampal volume is less than in panel $\mathbf{A}$, which shows the right hippocampal volume. The clinical report on this patient's original MRI (C) and Readers A and B correctly identified the patient's pathology.

gray matter-white matter contrast. In patients who are younger than 6 months, the amount of water content in the brain limits the gray matter-white matter differentiation, which is required for FreeSurfer to correctly delineate the brain anatomy. Thus, this technique cannot be accurately used to evaluate patients younger than 6 months.

The findings of this study have multiple limitations. There were only 24 pathologically confirmed cases of MTS included in our cohort of 57 patients. Such a small cohort may limit the statistical power; thus further studies performed in a larger sample are warranted. Also, the technique was not viable in patients with significant parenchymal volume loss due to postoperative changes, encephalomalacia, or infarct, or in patients younger than 6 months, which is a limiting factor in the indiscriminate use of this technique. We did not evaluate other pathologies that enlarge the hippocampus (such as cortical dysplasia) with this technique. A dedicated protocol would have to be in place, because there were 10 cases that could not be processed due to lack of preoperative or precontrast MPRAGE images.

\section{Conclusions}

Volumetric 3D imaging of the hippocampus is a valu-
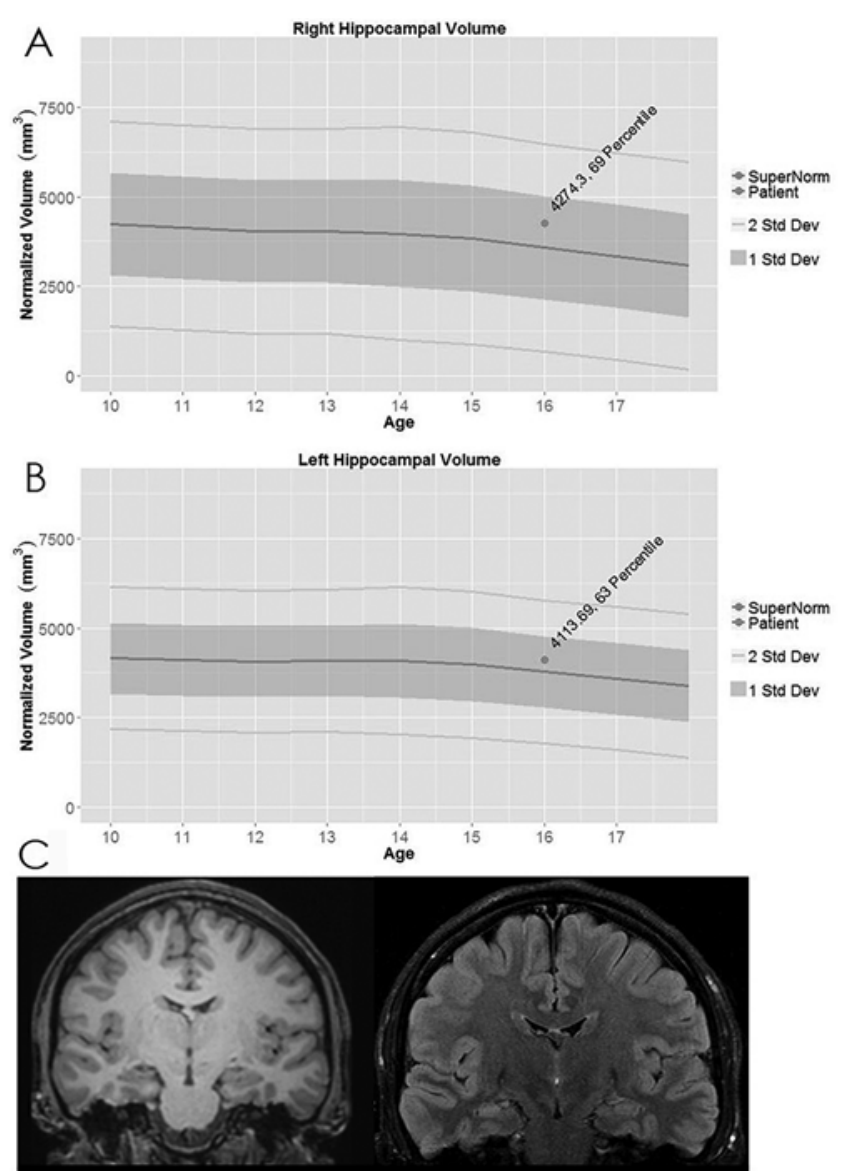

FIG. 6. Case 3. Right MTS proven on pathological investigation. Note that in panel $\mathbf{A}$, the right hippocampal volume is slightly more than in panel B, which shows the left hippocampal volume. This patient's original MRI (C) was incorrectly described as normal by all readers.

able tool for the identification of MTS that might be missed with anatomical imaging alone. Hippocampal volumetry is a reliable tool to identify MTS, especially in patients with subtle findings and no perceptible hippocampal atrophy on visual inspection. This method performs in a superior fashion across all measurements compared with a general reader, and it outperforms subspecialized neuroradiologists in sensitivity, PPV, and NPV. However, this tool is not reliable for use in patients with significant hemispheric atrophy or brain parenchymal absence as a result of prior brain injury from congenital or acquired causes, or in patients who are younger than 6 months. Further prospective studies involving a larger sample are warranted to confirm these findings.

\section{Acknowledgments}

The research described in this publication was supported by award no. U54 HD087011 from the Eunice Kennedy Shriver National Institute of Child Health and Human Development of the NIH, which was given to the Intellectual and Developmental Disabilities Research Center at Washington University. In addition, award no. PO1A903991 from the Healthy Aging and Senile Dementia Imaging Core and an unnumbered Barnes-Jewish Hospital Foundation grant support this research. Finally, Dr. Benzinger is a recipient of a grant from the National Multiple Sclerosis Society (PP1361). 
We thank Benjamin Speidel for his help retrieving the images, Amrita Hari-Raj for her help in creating Figure 3, and Veronica Vollmer for her help with figure production.

\section{References}

1. Akhondi-Asl A, Jafari-Khouzani K, Elisevich K, SoltanianZadeh H: Hippocampal volumetry for lateralization of temporal lobe epilepsy: automated versus manual methods. Neuroimage 54 (Suppl 1):S218-S226, 2011

2. Barkovich AJ, Kuzniecky RI: Neuroimaging of focal malformations of cortical development. J Clin Neurophysiol 13:481-494, 1996

3. Berkovic SF, McIntosh AM, Kalnins RM, Jackson GD, Fabinyi GCA, Brazenor GA, et al: Preoperative MRI predicts outcome of temporal lobectomy: an actuarial analysis. Neurology 45:1358-1363, 1995

4. Blümcke I, Thom M, Aronica E, Armstrong DD, Vinters HV, Palmini A, et al: The clinicopathologic spectrum of focal cortical dysplasias: a consensus classification proposed by an ad hoc Task Force of the ILAE Diagnostic Methods Commission. Epilepsia 52:158-174, 2011

5. Cleveland WS: Robust locally weighted regression and smoothing scatterplots. J Am Stat Assoc 74:829-836, 1979

6. Coan AC, Campos BM, Yasuda CL, Kubota BY, Bergo FP, Guerreiro CA, et al: Frequent seizures are associated with a network of gray matter atrophy in temporal lobe epilepsy with or without hippocampal sclerosis. PLoS One 9:e85843, 2014

7. Coan AC, Kubota B, Bergo FPG, Campos BM, Cendes F: 3T MRI quantification of hippocampal volume and signal in mesial temporal lobe epilepsy improves detection of hippocampal sclerosis. AJNR Am J Neuroradiol 35:77-83, 2014

8. Coan AC, Morita ME, Campos BM, Bergo FP, Kubota BY, Cendes F: Amygdala enlargement occurs in patients with mesial temporal lobe epilepsy and hippocampal sclerosis with early epilepsy onset. Epilepsy Behav 29:390-394, 2013

9. Engel J Jr, Van Ness PC, Rasmussen TB, Ojemann LM: Outcome with respect to epileptic seizures, in Engel J Jr (ed): Surgical Treatment of the Epilepsies, ed 2. New York: Raven Press, 1993

10. Free SL, Bergin PS, Fish DR, Cook MJ, Shorvon SD, Stevens JM: Methods for normalization of hippocampal volumes measured with MR. AJNR Am J Neuroradiol 16:637-643, 1995

11. Ghosh SS, Kakunoori S, Augustinack J, Nieto-Castanon A, Kovelman I, Gaab N, et al: Evaluating the validity of volumebased and surface-based brain image registration for developmental cognitive neuroscience studies in children 4 to 11 years of age. Neuroimage 53:85-93, 2010

12. Jack CR Jr, Sharbrough FW, Twomey CK, Cascino GD, Hirschorn KA, Marsh WR, et al: Temporal lobe seizures: lateralization with MR volume measurements of the hippocampal formation. Radiology 175:423-429, 1990

13. Jackson GD, Berkovic SF, Tress BM, Kalnins RM, Fabinyi GCA, Bladin PF: Hippocampal sclerosis can be reliably detected by magnetic resonance imaging. Neurology 40:18691875,1990

14. Jenkinson M, Bannister P, Brady M, Smith S: Improved optimization for the robust and accurate linear registration and motion correction of brain images. Neuroimage 17:825-841, 2002

15. Kasasbeh A, Hwang EC, Steger-May K, Bandt SK, Oberhelman A, Limbrick D, et al: Association of magnetic resonance imaging identification of mesial temporal sclerosis with pathological diagnosis and surgical outcomes in children following epilepsy surgery. J Neurosurg Pediatr 9:552-561, 2012

16. Keller SS, Richardson MP, O'Muircheartaigh J, SchoeneBake JC, Elger C, Weber B: Morphometric MRI alterations and postoperative seizure control in refractory temporal lobe epilepsy. Hum Brain Mapp 36:1637-1647, 2015

17. McIntosh AM, Wilson SJ, Berkovic SF: Seizure outcome after temporal lobectomy: current research practice and findings. Epilepsia 42:1288-1307, 2001

18. Meiners LC, van Gils A, Jansen GH, de Kort G, Witkamp TD, Ramos LM, et al: Temporal lobe epilepsy: the various MR appearances of histologically proven mesial temporal sclerosis. AJNR Am J Neuroradiol 15:1547-1555, 1994

19. Ng YT, McGregor AL, Duane DC, Jahnke HK, Bird CR, Wheless JW: Childhood mesial temporal sclerosis. J Child Neurol 21:512-517, 2006

20. Ng YT, McGregor AL, Wheless JW: Magnetic resonance imaging detection of mesial temporal sclerosis in children. Pediatr Neurol 30:81-85, 2004

21. Pardoe HR, Pell GS, Abbott DF, Jackson GD: Hippocampal volume assessment in temporal lobe epilepsy: How good is automated segmentation? Epilepsia 50:2586-2592, 2009

22. Russ SA, Larson K, Halfon N: A national profile of childhood epilepsy and seizure disorder. Pediatrics 129:256-264, 2012

23. Téllez-Zenteno JF, Hernández-Ronquillo L: A review of the epidemiology of temporal lobe epilepsy. Epilepsy Res Treat 2012:630853, 2012

24. Thom M: Review: Hippocampal sclerosis in epilepsy: a neuropathology review. Neuropathol Appl Neurobiol 40:520543,2014

25. Yang T, Zhou D, Stefan H: Why mesial temporal lobe epilepsy with hippocampal sclerosis is progressive: uncontrolled inflammation drives disease progression? J Neurol Sci 296:1-6, 2010

\section{Disclosures}

Dr. Benzinger discloses relationships outside of this work (i.e., participation in clinical trials) with Roche, Eli Lilly, and Avid Radiopharmaceuticals. She also receives support from Avid Radiopharmaceuticals (Eli Lilly) for a non-study-related clinical or research effort that she oversees. Dr. Matthew Smyth is cofounder of and a stock options owner in Therma Neurosciences, a company investigating the use of focal cooling devices for the treatment of epilepsy. He received no monetary compensation, and there is no conflict with this manuscript.

\section{Author Contributions}

Conception and design: Guzmán Pérez-Carrillo, Owen, Benzinger. Acquisition of data: Guzmán Pérez-Carrillo, Owen, Schwetye, McFarlane, Vellimana, Smyth. Analysis and interpretation of data: Guzmán Pérez-Carrillo, Owen, Schwetye, Miller-Thomas, Shimony, Benzinger. Drafting the article: Guzmán Pérez-Carrillo, Owen, Schwetye, Vellimana, Benzinger. Critically revising the article: all authors. Reviewed submitted version of manuscript: all authors. Approved the final version of the manuscript on behalf of all authors: Guzmán Pérez-Carrillo. Statistical analysis: Owen. Administrative/technical/material support: Guzmán PérezCarrillo. Study supervision: Guzmán Pérez-Carrillo, Mar, Smyth, Benzinger.

\section{Supplemental Information \\ Previous Presentations}

Portions of this work were presented as an educational exhibit at the 2015 American Society of Neuroradiology Annual Meeting in Chicago.

\section{Correspondence}

Gloria J. Guzmán Pérez-Carrillo, Advanced Neuro-Imaging Biometrics Initiative, Department of Medical Imaging, $1501 \mathrm{~N}$ Campbell Ave., Tucson, AZ 85724. email: gguzman@radiology. arizona.edu. 